\title{
Nonlinearly combined impacts of initial perturbation from human activities and parameter perturbation from climate change on the grassland ecosystem
}

\author{
G. Sun $^{1}$ and M. $\mathbf{M u}^{2,1}$ \\ ${ }^{1}$ The State Key Laboratory of Numerical Modeling for Atmospheric Sciences and Geophysical Fluid Dynamics (LASG), \\ Institute of Atmospheric Physics, Chinese Academy of Sciences, Beijing 100029, China \\ ${ }^{2}$ Key Laboratory of Ocean Circulation and Wave, Institute of Oceanology, Chinese Academy of Sciences, \\ Qingdao 266071, China
}

Received: 23 December 2010 - Revised: 12 October 2011 - Accepted: 12 October 2011 - Published: 29 November 2011

\begin{abstract}
Human activities and climate change are important factors that affect grassland ecosystems. A new optimization approach, the approach of conditional nonlinear optimal perturbation (CNOP) related to initial and parameter perturbations, is employed to explore the nonlinearly combined impacts of human activities and climate change on a grassland ecosystem using a theoretical grassland model. In our study, it is assumed that the initial perturbations and parameter perturbations are regarded as human activities and climate change, respectively. Numerical results indicate that the climate changes causing the maximum effect in the grassland ecosystem are different under disparate intensities of human activities. This implies the pattern of climate change is very critical to the maintenance or degradation of grassland ecosystem in light of high intensity of human activities and that the grassland ecosystem should be rationally managed when the moisture index decreases. The grassland ecosystem influenced by the nonlinear combination of human activities and climate change undergoes abrupt change, while the grassland ecosystem affected by other types of human activities and climate change fails to show the abrupt change under a certain range of perturbations with the theoretical model. The further numerical analyses also indicate that the growth of living biomass and the evaporation from soil surface shaded by the wilted biomass may be crucial factors contributing to the abrupt change of the grassland equilibrium state within the theoretical model.
\end{abstract}

\section{Correspondence to: G. Sun} (sungd@mail.iap.ac.cn)

\section{Introduction}

Since the industrial revolution, the global grassland ecosystems have suffered gigantic change. Chen et al. (2006) showed that grassland area stably decreased during 1860 1930 in the southern United States. Houghton and Hackler (2003) estimated that the grassland area decreased about $15 \%$ from 1850 to 2000 in China. Human activities and climate change are two important factors that cause the variations in grassland area and its biomass (Vitousek et al., 1997; White et al., 2000; Gibbard et al., 2005; Fay et al., 2008; Rogiers et al., 2008).

There have been many studies to explore how human activities and climate change influence the grasslands, such as degradation of the grasslands and its variations in carbon storage. The abrupt change from a grassland ecosystem to a desert ecosystem occurs as a result of intensive human activities, such as animal husbandry and grazing, and interannual climate variability. Zeng and Neelin (2000) proposed that the interannual climate variability tended to strong transition between forest and desert, thus favoring the holding of grasslike state. Sun and Mu (2009) displayed the nonlinear character of abrupt change from a grassland ecosystem to a desert ecosystem due to human activities. On the other hand, anthropogenic land use and climate change alter the carbon storage in grassland ecosystems, especially for carbon capacity of soils. Solomon et al. (2007) indicated that in the subtropical grassland ecosystems, the soil carbon content decreased, and that the grassland played a role in net carbon source. Mitchell and Csillag (2001) stated that climate variability affected the stability of productivity levels

Published by Copernicus Publications on behalf of the European Geosciences Union and the American Geophysical Union. 
and caused uncertainties in net primary product predictions in the northern mixed grass prairie. Although there are many research projects about the impact of human activities and climate change on grassland ecosystems, less studies have considered their combined impact on grasslands. McGuire et al. (2001) discussed the linearly combined responses of terrestrial ecosystem to land use and climate change using four ecosystem models and revealed their different roles in affecting the terrestrial ecosystem. However, the responses of terrestrial ecosystem to human activities and climate change are nonlinear, and the responses to different types and combinations of human activities and climate change vary. The investigation about which type of human activities and climate change leads to maximum effect on grassland ecosystems is important under the condition of grasslands management.

To explore which types of human activities and climate change bring maximum effect on grassland ecosystems, a conditional nonlinear optimal perturbation related to initial and parameter perturbations (CNOP, Mu et al., 2010) approach is employed as a nonlinear optimization method. The CNOP, which represents human activities and climate change within a reasonable range of perturbation, is a nonlinear initial and parameter perturbations approach. The CNOP can be regarded as representing the severest impacts of initial and parameter perturbations on grassland ecosystems. The approach has been employed in a previous study (Mu et al., 2010). In this work, a five-variable grassland ecosystem model (Zeng et al., 2006) is adopted in order to study the nonlinear combination of human activities and climate changes.

\section{Model, method and experimental design}

\subsection{Method and model}

The CNOP, whose nonlinear evolution attains the maximum value of a cost function under a certain constraint, is a type of perturbation related to initial conditions and parameters. Here, we will review how to obtain the CNOP. Let the nonlinear differential equations be as follows:

$$
\begin{aligned}
& \frac{\partial \boldsymbol{U}}{\partial t}=F(\boldsymbol{U}, \boldsymbol{P}) \quad \boldsymbol{U} \in R^{n}, t \in[0, T] \\
& \left.\boldsymbol{U}\right|_{t=0}=\boldsymbol{U}_{0},
\end{aligned}
$$

where $F$ is a nonlinear differential operator. $\boldsymbol{U}_{0}$ is an initial state vector and $\boldsymbol{P}$ is a model parameter vector. Let $M_{\tau}$ be the propagator of the Eq. (1) from the initial time 0 to the time $\tau . \boldsymbol{U}(\tau)$ is a solution of the Eq. (1) with the initial conditions $\boldsymbol{U}_{0}$ and the parameters $\boldsymbol{P}$ at time $\tau$ and satisfies $\boldsymbol{U}(\tau)=M_{\tau}\left(\boldsymbol{U}_{0}, \boldsymbol{P}\right)$.

Assuming there is an initial perturbation vector $\boldsymbol{u}_{0}$ and a parameter perturbation vector $\boldsymbol{p}$ with respect to $\boldsymbol{U}_{0}$ and $\boldsymbol{P}$, respectively, we obtain the solution to the Eq. (1) with the initial values $\boldsymbol{U}_{0}+\boldsymbol{u}_{0}$ and parameters $\boldsymbol{P}+\boldsymbol{p}$

$$
\boldsymbol{U}(\tau)+\boldsymbol{u}(\tau)=M_{\tau}\left(\boldsymbol{U}_{0}+\boldsymbol{u}_{0}, \boldsymbol{P}+\boldsymbol{p}\right),
$$

where $\boldsymbol{u}(\tau)$ depicts the divergence concerning the reference state $\boldsymbol{U}(\tau)$ due to the perturbations $\boldsymbol{u}_{0}$ and $\boldsymbol{p}$. For a chosen norm $\|\cdot\|$, a nonlinear combination of initial perturbation and parameter perturbation $\left(\boldsymbol{u}_{0 \delta}, \boldsymbol{p}_{\sigma}\right)$ is called a CNOP in terms of the following nonlinear optimization problem if and only if

$J\left(\boldsymbol{u}_{0 \delta}, \boldsymbol{p}_{\sigma}\right)=\max _{\boldsymbol{u}_{0} \in \Omega_{\delta}, \boldsymbol{p} \in \Omega_{\sigma}} J\left(\boldsymbol{u}_{0}, \boldsymbol{p}\right)$,

where

$J\left(\boldsymbol{u}_{0}, \boldsymbol{p}\right)=\left\|M_{\tau}\left(\boldsymbol{U}_{0}+\boldsymbol{u}_{0}, \boldsymbol{P}+\boldsymbol{p}\right)-M_{\tau}\left(\boldsymbol{U}_{0}, \boldsymbol{P}\right)\right\|$.

$\boldsymbol{u}_{0} \in \Omega_{\delta}$ and $\boldsymbol{p} \in \Omega_{\sigma}$ are constraint conditions. $J$ is a cost function.

The CNOP has two special cases introduced by $\mathrm{Mu}$ et al. (2010):

$J\left(\boldsymbol{u}_{0 \xi}\right)=\max _{\boldsymbol{u}_{0} \in \Omega_{\xi}} J\left(\boldsymbol{u}_{0}\right)$,

where

$J\left(\boldsymbol{u}_{0}\right)=\left\|M_{\tau}\left(\boldsymbol{U}_{0}+\boldsymbol{u}_{0}, \boldsymbol{P}\right)-M_{\tau}\left(\boldsymbol{U}_{0}, \boldsymbol{P}\right)\right\|$.

$\boldsymbol{u}_{0 \xi}$ is a CNOP that is related to the initial perturbations (CNOP-I) alone without the parameters perturbations. Moreover,

$J\left(\boldsymbol{p}_{\zeta}\right)=\max _{\boldsymbol{p} \in \Omega_{\zeta}} J(\boldsymbol{p})$,

where

$J(\boldsymbol{p})=\left\|M_{\tau}\left(\boldsymbol{U}_{0}, \boldsymbol{P}+\boldsymbol{p}\right)-M_{\tau}\left(\boldsymbol{U}_{0}, \boldsymbol{P}\right)\right\|$,

$\boldsymbol{p}_{\zeta}$ is another CNOP that is involved in parameter perturbations (CNOP-P) alone without the initial perturbations. $\boldsymbol{u}_{0} \in \Omega_{\xi}$ and $\boldsymbol{p} \in \Omega_{\zeta}$ are constraint conditions with respect to initial and parameter perturbations, and are the same as those in the Eq. (3) in our study.

From the above three optimization problems (3), (5) and (7), we find that $\left(\boldsymbol{u}_{0 \delta}, \boldsymbol{p}_{\sigma}\right)$ is a type of perturbation that causes the maximal value of the Eq. (4). The combination of $\boldsymbol{u}_{0 \xi}$ and $\boldsymbol{p}_{\zeta}$ is also a type of perturbation that causes the value of the Eq. (4) to distinctly be no larger than $\left(\boldsymbol{u}_{0 \delta}, \boldsymbol{p}_{\sigma}\right)$. It is seen that $\left(\boldsymbol{u}_{0 \delta}, \boldsymbol{p}_{\sigma}\right)$ is a nonlinear combination of initial perturbations and parameter perturbations, while $\left(\boldsymbol{u}_{0 \xi}, \boldsymbol{p}_{\zeta}\right)$ is a linear combination of initial and parameter perturbations.

To obtain the optimal value of the Eqs. (3), (5) and (7), the sequential quadratic programming (SQP) optimization algorithm (Barclay et al., 1998) is employed. In our study, $\mathrm{L}_{2}$ norm is applied and the constraint conditions of the initial perturbations and the parameter perturbations are $\left\|\boldsymbol{u}_{0}\right\| \leq \delta_{1}$ and $\|\boldsymbol{p}\| \leq \delta_{2}$ as a substitute for $\boldsymbol{u}_{0} \in \Omega_{\delta}, \boldsymbol{p} \in \Omega_{\sigma}, \boldsymbol{u}_{0} \in \Omega_{\xi}$ and $\boldsymbol{p} \in \Omega_{\zeta}$ in the Eqs. (3), (5) and (7). $\delta_{1}$ and $\delta_{2}$ measure the amplitude of the initial perturbations and the parameter perturbations. The larger $\delta_{1}$ and $\delta_{2}$ are, the higher intensities 
of the human activities and the climate change are according to our assuming corresponding relationship.

The five-variable grassland ecosystem model considers a single vertical column of soil and is related to one species of grass in Inner Mongolia (Zeng et al., 2005). It could analyze the hysteresis phenomenon over a large area of wellmixed grass in the arid and semi-arid regions. The seasonal variations in the grass and soil moisture are neglected. The model could deal with both the variations in the grass and soil moisture, as well as vegetation-soil interaction. The five state variables in the model are living biomass $\left(M_{\mathrm{c}}\right)$, wilted biomass $\left(M_{\mathrm{d}}\right)$, water content in the vegetation canopy $\left(W_{\mathrm{c}}\right)$, water content in the thin surface layer of soil $\left(W_{\mathrm{s}}\right)$, and water content in the rooting layer $\left(W_{\mathrm{r}}\right)$. Further details about the model parameters and their physical explanations can be found in the Appendix A. The variations in the grassland ecosystem imply the decreasing or increasing amounts of the living biomass, the wilted biomass, the soil moisture of the surface layer, and the soil moisture of the root zone by integrating the five-variable model. Moreover, the variations in the grassland ecosystem at the optimization time are measured with the Eq. (4), and called as the ecosystem perturbation.

\subsection{Experimental design}

To explore the maximum ecosystem perturbation due to the nonlinear combination of human activities and climate change, four state variables and the moisture index $\mu$ in the theoretical model are chosen. The $W_{\mathrm{c}}$ is not considered due to filtering the high frequency variations in the $M_{\mathrm{c}}$ and $M_{\mathrm{d}}$ (Appendix A). In our study, we assume that the initial perturbations in the amounts of the living biomass, the wilted biomass, the soil moisture of the surface layer and the soil moisture of the root zone, as well as the parameter perturbation in the moisture index $\mu$, represent human activities and climate change, respectively. The decreasing or increasing in the living biomass, the wilted biomass, and the soil moisture in the two layers are considered results of human activity such as animal husbandry, grazing, digging groundwater, irrigation and so on, though their variations may result from climate change.

The moisture index $\mu$ is an important parameter in the theoretical model and prescribes climate condition. The definition of the moisture index is $\mu=\frac{\text { Prec }}{e_{\mathrm{s}}^{*}}$. Prec and $e_{\mathrm{s}}^{*}$ denote the annual precipitation and the annual maximal potential evaporation from the soil surface layer. We introduce the moisture index in Appendix A. The variation in the moisture index represents the climate change in our studies. In fact, climate change is also caused by human activities. In our work, the moisture index change due to human activities is not considered. It is well known to us that the climate condition is variational. In this study, the perturbations of the annual moisture index are considered and organized as a parameter perturbation vector. The annual perturbation is superimposed on the annual moisture index to introduce variations in the moisture index. In so doing, the parameter $\mu$ should be optimized year by year in order to estimate interannual moisture index change. The aim lies in exploring the response of the grassland ecosystem to the evolution of the moisture index with time. When the five-variable grassland model is discretized according to the time step, the moisture index is optimized step by step in every time step to exhibit the climate change. Accordingly, the vector $\boldsymbol{P}$ is $\left(\mu_{1}, \mu_{2}, \cdots, \mu_{n}\right)$, and its perturbation vector $\boldsymbol{p}=\left(\mu_{1}^{\prime}, \mu_{2}^{\prime}, \cdots, \mu_{n}^{\prime}\right) . n$ is the dimension of the parameter perturbation vector, corresponding to the number of optimization steps as well.

The variations in the grassland at the final time are characterized by the Eq. (4) for the four state variables by integrating the five-variable grassland model. The grassland ecosystem perturbations at the optimization time due to nonlinear and linear combinations of the human activities and the climate changes are clearly depicted by $J_{1}$ and $J_{2}$, called ecosystem perturbations:

$J_{1}\left(\boldsymbol{u}_{0}, \boldsymbol{p}\right)=\left\|M_{\tau}\left(\boldsymbol{U}_{0}+\boldsymbol{u}_{0 \delta}, \boldsymbol{P}+\boldsymbol{p}_{\sigma}\right)-M_{\tau}\left(\boldsymbol{U}_{0}, \boldsymbol{P}\right)\right\|$.

$J_{2}\left(\boldsymbol{u}_{0}, \boldsymbol{p}\right)=\left\|M_{\tau}\left(\boldsymbol{U}_{0}+\boldsymbol{u}_{0 \xi}, \boldsymbol{P}+\boldsymbol{p}_{\zeta}\right)-M_{\tau}\left(\boldsymbol{U}_{0}, \boldsymbol{P}\right)\right\|$.

$\boldsymbol{u}_{0 \delta}$ and $\boldsymbol{p}_{\sigma}$ are obtained by optimizing the Eq. (3), while $\boldsymbol{u}_{0 \xi}$ and $\boldsymbol{p}_{\zeta}$ are calculated by optimizing the Eqs. (5) and (7). The grassland equilibrium state (GES) values - which are 0.553 for the living biomass, 0.641 for the soil moisture of surface layer, 0.635 for the soil moisture of root zone and 0.580 for the wilted biomass by integrating the five-variable grassland ecosystem model when the moisture index $\mu$ is 0.31 and the other parameters referring to the appendix A are fixed - function as the initial values of the reference state. According to previous research (Liu and Gao, 2008), the amplitude $\delta_{2}$ is chosen as 0.22 , which is in agreement with results based on observational results when the annual perturbation is superimposed on the annual moisture index (details in Appendix $\mathrm{B})$. The larger amplitude $\left(\delta_{2}=0.35\right)$ is also applied to explore the ecosystem perturbation under decreasing of the moisture index. The amplitude $\delta_{1}$ of initial perturbation is $0.0,0.1,0.2,0.26,0.3$ and 0.4 to explore the sensitivity of the grassland ecosystem to different human activity intensities. The amplitudes account for about 5\% 30\% of the normalized initial grassland state. The optimization time is $20 \mathrm{yr}$. The model is discretized based on the fourth-order Runge-Kutta method with a time step of $d t=1 / 24$ (representing half of one month).

To explore the impacts of nonlinear combination of human activities and climate change on the grassland ecosystem, the initial perturbation and the parameter perturbation are, respectively, optimized alone. The nonlinear evolutions of the grassland ecosystem influenced by the linear combination of the initial perturbation and the parameter perturbation optimized alone are exhibited to discuss the final state for the living biomass, the wilted biomass and the soil moisture in the 


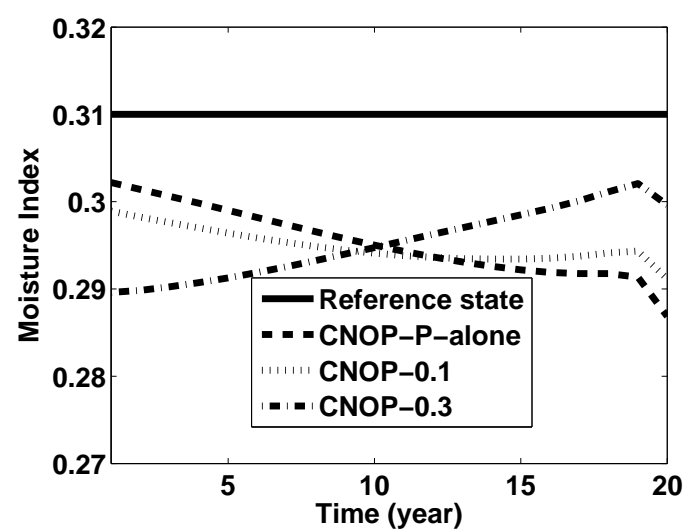

Fig. 1. The variations in the moisture index when $\delta_{2}=0.22$ and the annual perturbations have been superimposed on the reference moisture index. CNOP-P-alone implies the moisture index as a parameter being optimized the Eq. (7) alone. CNOP-0.1 and CNOP0.3 imply the parameter perturbation of the CNOP obtained when $\delta_{1}=0.1$ and $\delta_{1}=0.3$ by optimizing the Eq. (3).

surface layer and root zone. Additionally, we run the model with the ecosystem equilibrium state as the initial value and $\mu$ superimposed by parameter perturbations during the optimization time. Afterward, the model is run successively with the reference state of the moisture index to consider the nonlinear evolution of the perturbed ecosystem.

\section{Numerical results}

\subsection{CNOP}

In this section, the patterns of the moisture index under different constraint parameters related to the initial condition are compared. Figure 1 shows that the perturbed moisture index, which is that the parameter perturbations are superimposed on the original moisture index, continually decreases due to the parameter perturbations when the parameter $\mu$ is optimized alone and $\delta_{2}=0.22$. When $\delta_{1}=0.1$, which represents the intensity of the human activities, and $\delta_{2}=0.22$, which represents the intensity of the climate change, the perturbed moisture index also continually decreases. The results imply that the differences between the variations in the moisture index with the weak intensity of human activities and without the human activities are small. However, when the intensity of human activities increases, the perturbed moisture index that leads to the maximal ecosystem perturbation is different. For example, when $\delta_{1}=0.3$ and $\delta_{2}=0.22$, the perturbed moisture index seriously decreases during the initial optimization time. The perturbed moisture index continually increases and weakly decreases in the last optimization time. The numerical results demonstrate that the decreasing of the moisture index may be a key factor leading to the

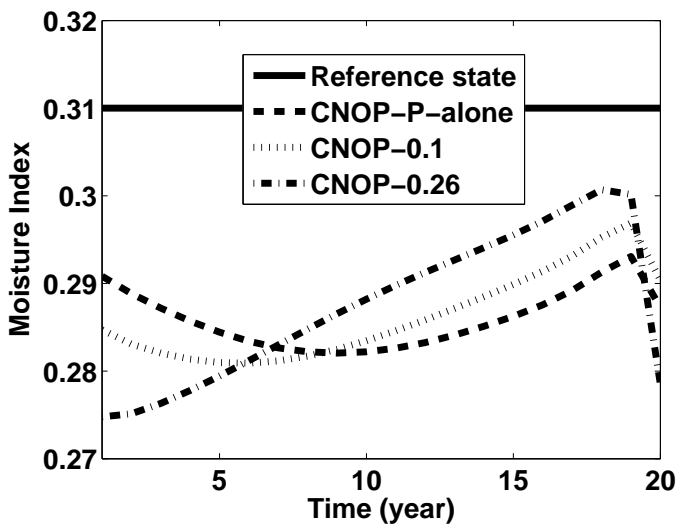

Fig. 2. Same as in Fig. 1, but for $\delta_{2}=0.35$.

maximal ecosystem perturbation over the initial time when the intensity of human activities increases.

The CNOP is also calculated when $\delta_{2}=0.35$, representing the stronger climate change (Fig. 2). The variations of the moisture index are similar between without and with considering the human activities $\left(\delta_{1}=0.1\right)$. With the increasing of $\delta_{1}$, the variations of the moisture index are different, especially during the initial time. However, the CNOPs related to the initial perturbation are similar among $\delta_{2}=0.22,0.35$ and the initial perturbation being optimized alone (Table 1).

\subsection{Nonlinear evolution of the grassland ecosystem for different optimization results}

In the above results, the CNOP-I optimized alone for the initial perturbation representing only human activities, the CNOP-P optimized alone for the moisture index $\mu$ indicating only climate change, and the CNOP optimized simultaneously to represent the nonlinear combination of human activities and climate change are shown. The incorporation of the optimal initial perturbations optimized by the Eq. (5) and the optimal parameter perturbations optimized by the Eq. (7) are called the linear combination of human activities and climate change. To explore the impact of the nonlinear combination of human activities and climate change on the grassland ecosystem, the nonlinear ecosystem perturbation and evolutions of the grassland ecosystem are exhibited for the different optimization results (Table 2 and Fig. 3) under moderate climatic change $\delta_{2}=0.22$. When $\delta_{1}=0.1$ and $\delta_{2}=0.22$, the ecosystem perturbation and evolutions of the grassland ecosystem influenced by the nonlinear and linear combinations of human activities and climate change are similar. For example, the levels of the resulting perturbation of the grassland ecosystem are 0.317 and 0.315 (see Table 2), corresponding to the nonlinear and linear combinations of human activities and climate change, respectively. With the increasing of $\delta_{1}$, the ecosystem perturbations are augmented and the difference between linear and nonlinear 
Table 1. The CNOP-Is for different constraint conditions $\delta_{1}$.

\begin{tabular}{llll}
\hline$\delta_{1}$ & Alone $\left(\times 10^{-2}\right)$ & $0.22\left(\times 10^{-2}\right)$ & $0.35\left(\times 10^{-2}\right)$ \\
\hline 0.1 & $(-5.50,-6.68,-4.37,-7.08)$ & $(-5.48,-6.76,-4.43,-7.06)$ & $(-5.45,-6.88,-4.50,-7.03)$ \\
0.2 & $(-11.76,-1.25,-8.40,-13.77)$ & $(-11.70,-1.28,-8.57,-13.71)$ & $(-11.64,-1.31,-8.76,-13.65)$ \\
0.3 & $(-19.23,-1.73,-11.90,-19.63)$ & $(-19.12,-1.78,-12.26,-19.52)$ & $(-19.03,-1.83,-12.54,-19.43)$ \\
0.4 & $(-29.36,-2.00,-14.00,-23.19)$ & $(-29.18,-2.08,-14.55,-23.07)$ & $(-29.08,-2.12,-14.82,-23.03)$ \\
\hline
\end{tabular}

Note: the second column "Alone" represents the human activities is considered without climate change by optimizing the Eq. (5). The third and fourth columns are the component of the CNOP related to initial perturbation for different $\delta_{2}$ by optimizing the Eq. (3). The values in the bracket are the initial perturbations for the living biomass, the soil moisture in the surface layer, the soil moisture of the root zone and the wilted biomass.
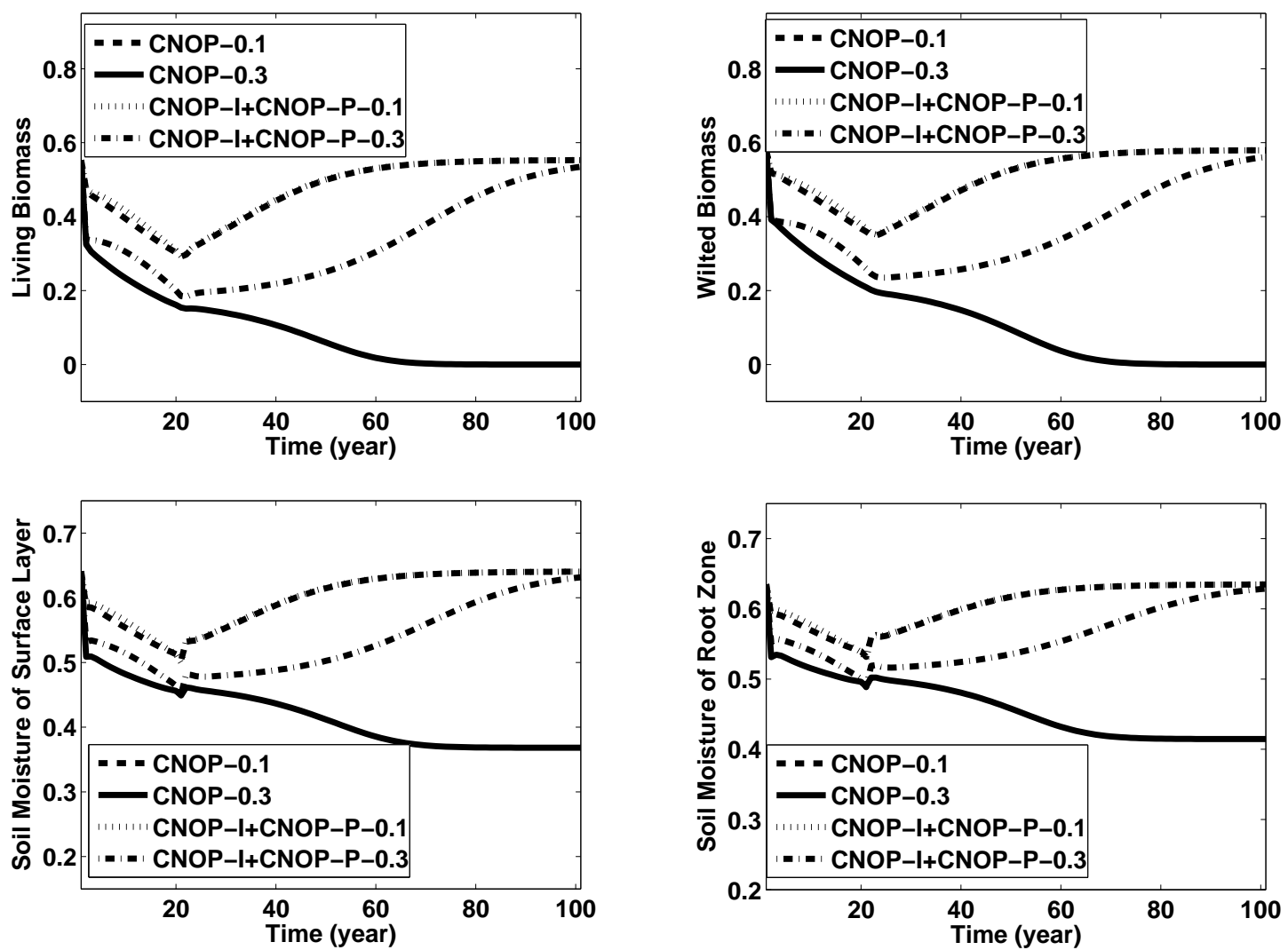

Fig. 3. The nonlinear evolutions in grassland ecosystems for different CNOPs when $\delta_{2}=0.22$. CNOP-0.1 implies that the grassland ecosystem is caused by the perturbations obtained by optimizing the Eq. (3) when $\delta_{1}=0.1$ and $\delta_{2}=0.22$. CNOP-0.3 is same as CNOP-0.1, only when $\delta_{1}=0.3$. CNOP-I+CNOP-P-0.1 implies that the grassland ecosystem is caused by the perturbations obtained by optimizing the Eqs. (5) and (7) when $\delta_{1}=0.1$ and $\delta_{2}=0.22$. CNOP-I+CNOP-P-0.3 is same as CNOP-I+CNOP-P-0.1, only when $\delta_{1}=0.3$. The living biomass (top left), the wilted biomass (top right), the soil moisture of surface layer (bottom left), and the soil moisture of root zone (bottom right).

contributions also increases. For example, when $\delta_{1}=0.4$ and $\delta_{2}=0.22$, the ecosystem perturbations are 0.629 and 0.575 (obtained by the Eq. 4) due to the nonlinear and linear combination of human activities and climate change. At the same time, the grassland ecosystem influenced by the linear combination of human activities and climate change comes back to the initial ecosystem state when $\delta_{1}=0.3$ and $\delta_{2}=0.22$. However, the grassland ecosystem influenced by the nonlinear combination of human activities and climate change evolves to a desert ecosystem, whose living biomass and wilted biomass become zero. The living biomass, the wilted biomass and the soil moisture in the two layers rapidly decrease during the initial period due to human activity. In the following years, the living biomass, the wilted biomass and the soil moisture in the two layers gradually are reduced due to the combined effect of human activity and climate 

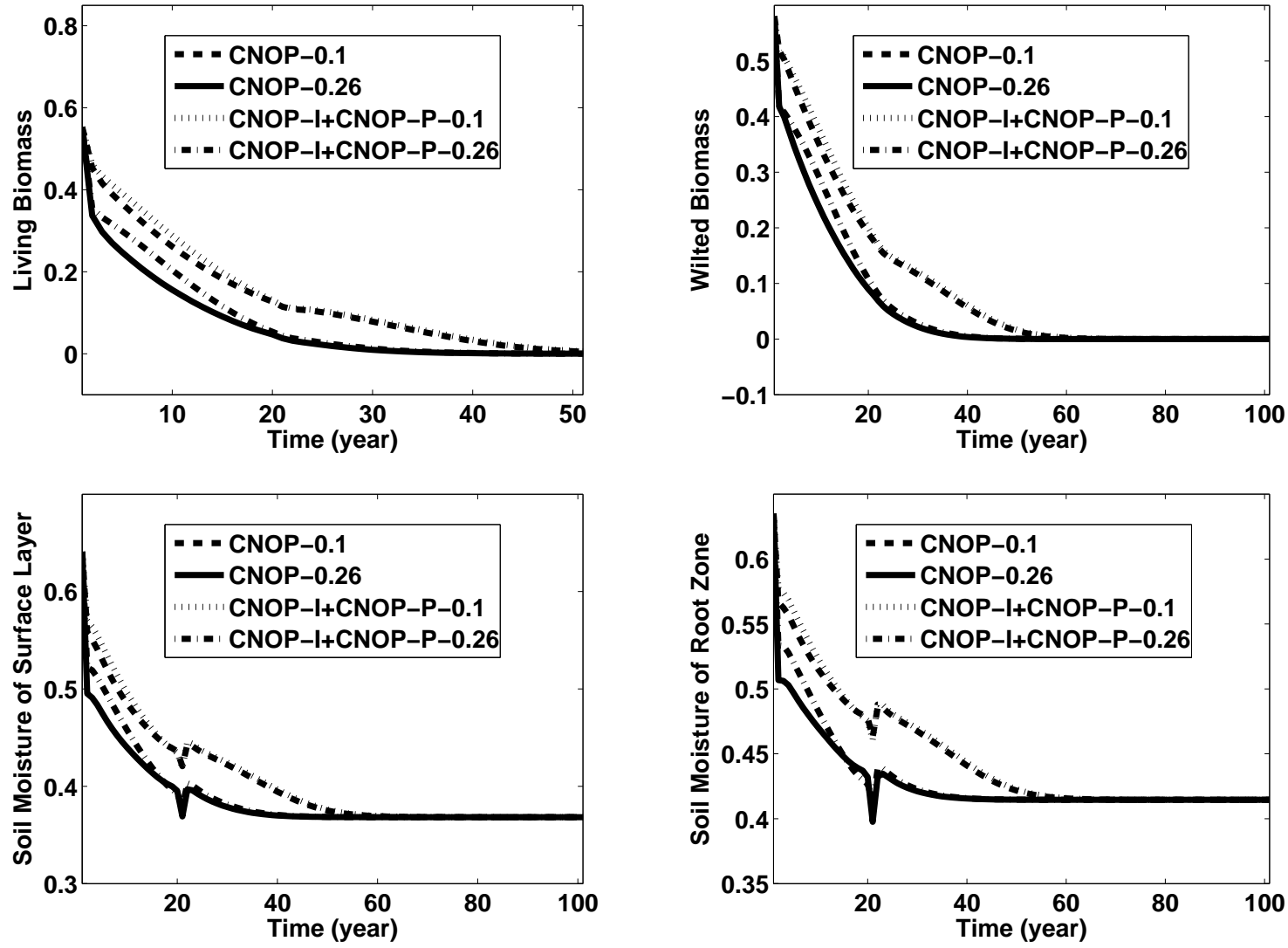

Fig. 4. Same as in Fig. 3, but for $\delta_{2}=0.35$. The living biomass (top left), the wilted biomass (top right), the soil moisture of surface layer (bottom left), and the soil moisture of root zone (bottom right).

Table 2. The variations calculated by the Eq. (4) in the grassland ecosystem caused by the CNOP obtained by optimizing the Eq. (3) and linear combination of the CNOP-I and the CNOP-P obtained by optimizing the Eqs. (5) and (7).

\begin{tabular}{ccc}
\hline$\delta_{1}$ & CNOP & Linear \\
\hline 0.1 & 0.317 & 0.315 \\
0.2 & 0.382 & 0.367 \\
0.3 & 0.494 & 0.451 \\
0.4 & 0.629 & 0.575 \\
\hline
\end{tabular}

change. Finally, the living biomass and the wilted biomass evolve to zero. The soil moisture in the two layers decrease to minimum levels.

Figure 4 shows the same diagnostics as Fig. 3, but for $\delta_{2}=0.35$. There are tiny differences concerning the evolutions of the grassland ecosystem due to the distinct intensities of human activities $\left(\delta_{1}=0.1\right.$ and 0.26$)$ when the intensity of climate change increases $\left(\delta_{2}=0.35\right)$. Although the grassland ecosystems influenced by the nonlinear and linear combination of human activities and climate change evolve to a desert ecosystem, the time for the living biomass and the wilted biomass to reach zero is shorter for the nonlinear combination of human activities and climate change in comparison with their linear combination (Fig. 4).

\subsection{Further analyses}

To explore why there are different responses of grassland ecosystem to the linear and nonlinear combination of human activities and climate change, we analyze the variations of physical processes in the grassland dynamics. According to the amplitude of variations in different physical processes, the growth of living biomass $G$ and the evaporation from soil surface shaded by the wilted biomass $E_{\mathrm{s}}$ are discussed. Figure 5 shows that, although the variations in $G$ and $E_{\mathrm{s}}$ caused by the nonlinear combination are smaller than those caused by the linear combination during the initial period, $G$ rapidly decreases and $E_{\mathrm{s}}$ rapidly increases in the following years. After several years, especially for the optimization time $(20 \mathrm{yr})$, the variations in $G$ and $E_{\mathrm{s}}$ caused by the nonlinear combination are greater than those caused by the linear combination. Finally, the abrupt change from the grassland equilibrium state into the desert equilibrium state occurs. The numerical results suggest that the physical processes $G$ and $E_{\mathrm{s}}$ play a key role in the ecosystem 

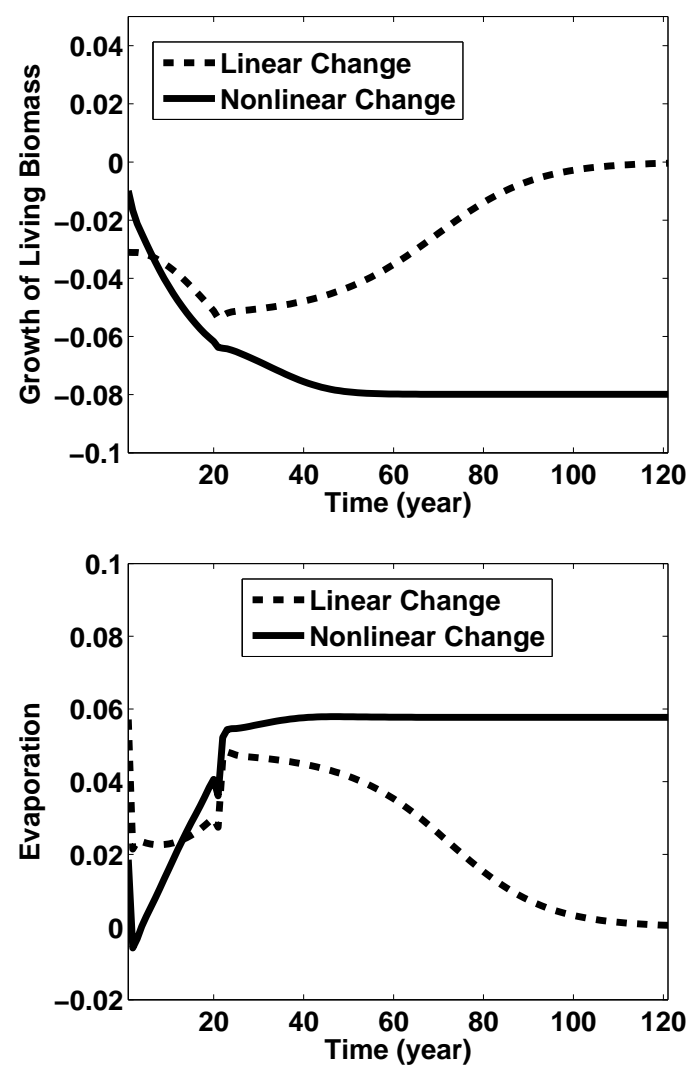

Fig. 5. The variations of the growth of living biomass $G$ and the evaporation from soil surface shaded by the wilted biomass $E_{\mathrm{S}}$ caused by different types human activities and climate change when $\delta_{1}=0.3$ and $\delta_{2}=0.22$. The growth of living biomass (top), and the evaporation (bottom).

perturbation. Their variations may be the important factors causing abrupt change of the grassland equilibrium state. The findings also validate the conclusions about the shading effects of the wilted biomass on the grassland equilibrium state proposed by Mu and Wang (2007).

\section{Model results and their linkage to real world}

The alteration of the grassland ecosystem is dominated by not only climate change and human activities discretely, but by their interaction and combination (Xu et al., 2009). For example, Gao et al. (2003) stated that the simulated area of grassland in north-south transect of eastern China with land use was smaller than that without land use under contemporary climate conditions using a ecosystem model. However, it is not yet explored which pattern of combination of human activities and climate change cause the severest effect on the grassland. In our work, the pattern is attempted to be shown although a simple model is employed. In terms of the observation data, the combined effect of human activities and climate change on the grassland is notable. For example,
Guo et al. (2008) employed satellite observation and indicated that the Talatan area incurred lawn desertification because of the warm and arid climate condition and unreasonable human activities. The area of the severe desertification increased $1.09 \times 10^{2} \mathrm{~km}^{2}$ from 1987 to 1996 . The evaporation increased and the precipitation slightly increased. This implied the decreasing of the moisture index $\mu=\operatorname{Prec} / e_{\mathrm{s}}^{*}$. At the same time, overgrazing and reclamation accelerated the lawn desertification. The findings show that grassland is easily destroyed due to decreases in the moisture index and unreasonable human activities, which is similar to our results. Zhao et al. (2008) found that the grassland coverage would decrease to zero facing the high grazing using a field grazing experimentation in China; even medium and light grazing would cause the desertification of the grassland. This may be because the climate condition is not bad enough to result in the desertification. The desertification of the grassland is a nonlinear physical process. The above observation results show that the desertification of the grassland is a result of the combination of the climate change and the human activities. Our findings also suggest that the nonlinear combination of climate change and human activities should be noticed during the nonlinear physical process of the grassland, especially for the desertification of the grassland. The linear combination of climate change and human activities should be avoided, as far as possible, to supply useful information for the policymakers. These interpretations must be taken with some cautions since the model used here is theoretical and thus provides a simplified dynamics, and since its ability to simulate real grassland ecosystems cannot be estimated. Interpretations can thus only be indicative and partially significant.

\section{Summary and conclusion}

Our work finds that the type of climate change is pivotal when there are different intensities of human activities, especially for the initial period. The nonlinearly combined impact of human activities and climate change plays an important role in the ecosystem perturbation, compared with their linearly combined impacts. For example, an abrupt change occurs and the timing of the abrupt change is earlier when the grassland ecosystems are influenced by the nonlinear combination of human activities and climate change, while the abrupt change fails to show for their linear combination. This research suggests that the CNOP approach is a useful tool to find the nonlinear combination of human activities and climate change that induces the maximum ecosystem perturbation within the theoretical model.

Our study is based on the hypothesis that the initial perturbation and the parameter perturbation are considered as human activities and climate change. However, the variations in the living biomass, the wilted biomass and the soil moisture may also be affected by climate change. At the same 
time, the variations in the moisture index may be caused by human activities. The above results also are founded on the theoretical model. The impacts of multi-factor on the grassland ecosystem will be discussed in a future study. There are different responses of the terrestrial ecosystem to human activities and climate change. McGuire et al. (2001) indicated that the effect of climate change on carbon storage was smaller than that of land use using four process-based ecosystem models. In our research, such comparisons are not made. We explored the nonlinearly combined effects of human activities and climate change on the grassland ecosystem. In addition, because the theoretical model is applied in this study, the impacts of increasing $\mathrm{CO}_{2}$ or its fertilization of the grassland ecosystem cannot be considered. There are many complex dynamic global vegetation models (DGVMs) proposed to explore the variations in plant types and carbon cycle due to increasing $\mathrm{CO}_{2}$, climate change and $\mathrm{N}$ deposition. It is interesting to discuss the influences of human activities, climate change and increasing $\mathrm{CO}_{2}$ on the terrestrial ecosystem employing DGVMs. These research topics will be explored in future work.

\section{Appendix A}

\section{The five-variable ecosystem model}

The five-variable grassland ecosystem model is given as follows (Zeng et al., 2006; Sun and Mu, 2009):

$$
\begin{aligned}
\frac{d M_{\mathrm{c}}}{d t}= & \alpha^{*}\left(G\left(M_{\mathrm{c}}, W_{\mathrm{r}}\right)-D_{\mathrm{c}}\left(M_{\mathrm{c}}, W_{\mathrm{r}}\right)-C_{\mathrm{c}}\left(M_{\mathrm{c}}\right)\right), \\
\frac{d M_{d}}{d t}= & \alpha^{*}\left(\beta^{\prime} D_{\mathrm{c}}\left(M_{\mathrm{c}}, W_{\mathrm{r}}\right)-D_{\mathrm{d}}\left(M_{\mathrm{d}}\right)-C_{\mathrm{d}}\left(M_{\mathrm{d}}\right)\right), \\
\frac{d W_{\mathrm{c}}}{d t}= & P_{\mathrm{c}}\left(M_{\mathrm{c}}\right)+E_{\mathrm{r}}\left(M_{\mathrm{c}}, W_{\mathrm{r}}\right) \\
& -E_{\mathrm{c}}\left(M_{\mathrm{c}}, W_{\mathrm{r}}\right)-R_{\mathrm{c}}\left(M_{\mathrm{c}}\right),
\end{aligned}
$$

$$
\begin{aligned}
\frac{d W_{s}}{d t}= & P_{\mathrm{s}}\left(M_{\mathrm{c}}\right)-E_{\mathrm{s}}\left(M_{\mathrm{c}}, W_{\mathrm{s}}, M_{\mathrm{d}}\right)+R_{\mathrm{c}}\left(M_{\mathrm{c}}\right) \\
& -Q_{\mathrm{sr}}\left(W_{\mathrm{s}}, W_{\mathrm{r}}\right)-R_{\mathrm{s}}\left(M_{\mathrm{c}}, W_{\mathrm{s}}, M_{\mathrm{d}}\right)
\end{aligned}
$$

$$
\begin{aligned}
\frac{d W_{\mathrm{r}}}{d t}= & P_{\mathrm{r}}\left(M_{\mathrm{c}}\right)+\alpha_{\mathrm{r}} R_{\mathrm{s}}\left(M_{\mathrm{c}}, W_{\mathrm{s}}, M_{\mathrm{d}}\right) \\
& -E_{\mathrm{r}}\left(M_{\mathrm{c}}, W_{\mathrm{r}}\right)+Q_{\mathrm{sr}}\left(W_{\mathrm{s}}, W_{\mathrm{r}}\right)-R_{\mathrm{r}}\left(M_{\mathrm{c}}, W_{\mathrm{r}}\right) .
\end{aligned}
$$

The meanings of the above equation terms are shown in Table A1. Their mathematical formulations are not shown but can be obtained in Zeng et al. (2006) and Sun and $\mathrm{Mu}$ (2009). Because Zeng et al. (2006) considered to filter the high frequency variations in $M_{\mathrm{c}}$ and $M_{\mathrm{d}}$, it was assumed that the equation $\frac{d W_{\mathrm{c}}}{d t}=P_{\mathrm{c}}\left(M_{\mathrm{c}}\right)+E_{\mathrm{r}}\left(M_{\mathrm{c}}, W_{\mathrm{r}}\right)-$
Table A1. The right terms in the five-variable ecosystem model.

\begin{tabular}{ll}
\hline $\begin{array}{l}\text { Right } \\
\text { terms }\end{array}$ & Meaning \\
\hline$G$ & the growth of the living biomass \\
$D_{\mathrm{c}}$ & the wilting of the living biomass \\
$C_{\mathrm{c}}$ & the consumption of the living biomass \\
$D_{\mathrm{d}}$ & the decomposition of the wilted biomass \\
$C_{\mathrm{d}}$ & the consumption of the wilted biomass \\
$P_{\mathrm{C}}$ & the precipitation reserved in the vegetation \\
& canopy \\
$P_{\mathrm{S}}$ & the precipitation reserved in the surface layer of \\
& soil \\
$P_{\mathrm{r}}$ & the precipitation reserved in the rooting layer \\
$E_{\mathrm{C}}$ & the evaporation from the vegetation canopy \\
$E_{\mathrm{S}}$ & the evaporation from the surface layer of soil \\
$E_{\mathrm{r}}$ & the evaporation from the rooting layer \\
$R_{\mathrm{C}}$ & the transpiration from the vegetation canopy \\
$R_{\mathrm{S}}$ & the transpiration from the surface layer of soil \\
$R_{\mathrm{r}}$ & the transpiration from the rooting layer \\
$Q_{\mathrm{sr}}$ & the conductive transport term between the sur- \\
& face layer of soil and the root zone
\end{tabular}

$E_{\mathrm{c}}\left(M_{\mathrm{c}}, W_{\mathrm{r}}\right)-R_{\mathrm{c}}\left(M_{\mathrm{c}}\right)$ was in balance, that is, $P_{\mathrm{c}}\left(M_{\mathrm{c}}\right)+$ $E_{\mathrm{r}}\left(M_{\mathrm{c}}, W_{\mathrm{r}}\right)-E_{\mathrm{c}}\left(M_{\mathrm{c}}, W_{\mathrm{r}}\right)-R_{\mathrm{c}}\left(M_{\mathrm{c}}\right)=0$. Therefore, the fivevariable grassland ecosystem model is simplified to only discuss the living biomass, the wilted biomass and the soil moisture in the surface layer and root zone. The Eqs. (A1-A5) do not explicitly include the parameter $\mu$. To clarify it, we introduce how to obtain the parameter in the model. The terms $P_{\mathrm{S}}$ and $P_{\mathrm{r}}$ are related to the annual precipitation Prec. The terms $E_{\mathrm{s}}$ and $E_{\mathrm{r}}$ are associated with the annual maximal potential evaporation $e_{\mathrm{s}}^{*}$ from the soil surface layer. Their expressions are:

$$
\begin{aligned}
& P_{\mathrm{c}}=\alpha_{\mathrm{c}} \operatorname{Prec}\left(1-e^{-\varepsilon_{\mathrm{c}} M_{\mathrm{c}} / M_{\mathrm{c}}^{*}}\right), \\
& P_{\mathrm{S}}=\alpha_{\mathrm{S}}\left(\operatorname{Prec}-P_{\mathrm{c}}\right), \\
& P_{\mathrm{r}}=\left(1-\alpha_{\mathrm{s}}\right)\left(\operatorname{Prec}-P_{\mathrm{c}}\right), \\
& E_{\mathrm{s}}=e_{\mathrm{s}}^{*} e^{-\epsilon_{\mathrm{Esz}} M_{\mathrm{d}} / M_{\mathrm{d}}^{*}}\left[e^{-\epsilon_{\mathrm{f}} M_{\mathrm{c}} / M_{\mathrm{c}}^{*}}\right. \\
& \left.+\left(1-e^{-\epsilon_{\mathrm{f}} M_{\mathrm{c}} / M_{\mathrm{c}}^{*}}\right)\left(1-\kappa_{1}\left(1-e^{-\epsilon_{\mathrm{Esx}} M_{\mathrm{c}} / M_{\mathrm{c}}^{*}}\right)\right)\right] \\
& \left(1-e^{-\epsilon_{\mathrm{Esy}_{1}} W_{\mathrm{s}} / W_{\mathrm{s}}^{*}}\right) \text {, } \\
& E_{\mathrm{r}}=e_{\mathrm{s}}^{*} \phi_{\mathrm{rs}}\left(1-e^{-\epsilon_{\mathrm{f}} M_{\mathrm{c}} / M_{\mathrm{c}}^{*}}\right)\left(1-\kappa_{\mathrm{Er}} e^{-\epsilon_{\mathrm{Erx}} M_{\mathrm{c}} / M_{\mathrm{c}}^{*}}\right) \\
& \left(1-e^{-\epsilon_{\operatorname{Ery}_{2}} W_{\mathrm{r}} / W_{\mathrm{r}}^{*}}\right) \text {, } \\
& R_{\mathrm{S}}=\lambda_{\mathrm{rs}} P_{S} e^{-\epsilon_{\mathrm{Rsz}} M_{\mathrm{d}} / M_{\mathrm{d}}^{*}}\left[e^{-\epsilon_{\mathrm{f}} M_{\mathrm{c}} / M_{\mathrm{c}}^{*}}\right. \\
& \left.+\left(1-e^{-\epsilon_{\mathrm{f}} M_{\mathrm{c}} / M_{\mathrm{c}}^{*}}\right)\left(1-\kappa_{\mathrm{Rs}}\left(1-e^{-\epsilon_{\mathrm{Rsx}} M_{\mathrm{c}} / M_{\mathrm{c}}^{*}}\right)\right)\right] \\
& \left(e^{\epsilon_{\mathrm{Rsy}} W_{\mathrm{s}} / W_{\mathrm{s}}^{*}}-1\right) \text {, }
\end{aligned}
$$


Table A2. The key parameter values in the five-variable ecosystem model.

\begin{tabular}{|c|c|c|}
\hline PA & VA & Meaning \\
\hline$M_{\mathrm{c}}^{*}$ & 0.1 & the characteristic value of the living biomass \\
\hline$M_{\mathrm{d}}^{*}$ & 0.1 & the characteristic value of the wilted biomass \\
\hline$W_{\mathrm{s}}^{*}$ & 40 & the characteristic value of the soil moisture of surface layer \\
\hline$W_{\mathrm{r}}^{*}$ & 200 & the characteristic value of the soil moisture of root zone \\
\hline$\alpha^{*}$ & 0.4 & the maximum growth rate \\
\hline$\beta^{\prime}$ & 0.1 & the characteristic wilting rate \\
\hline$\alpha_{\mathrm{s}}$ & 0.85 & coefficients about loss due to the surface layer in term $P_{\mathrm{S}}$ \\
\hline$\varepsilon_{\mathrm{c}}$ & 1.0 & exponential attenuation coefficients about the living biomass in term $P_{\mathrm{c}}$ \\
\hline$\alpha_{\mathrm{c}}$ & 0.05 & coefficients about loss due to the living biomass in term $P_{\mathrm{c}}$ \\
\hline$\alpha_{\mathrm{r}}$ & 0.1 & coefficients about the surface runoff inflow the root zone \\
\hline$e_{\mathrm{S}}^{*}$ & 1000 & the potential evaporation from the bare soil \\
\hline$\epsilon_{\mathrm{Esz}}$ & 1.0 & exponential attenuation coefficients about the wilted biomass in term $E_{\mathrm{S}}$ \\
\hline$\epsilon_{\mathrm{f}}$ & 200 & the parameter of the fraction of living grass coverage \\
\hline$\kappa_{1}$ & 0.4 & the parameter describing the vegetation-soil interaction \\
\hline$\epsilon_{\text {Esx }}$ & 0.7 & exponential attenuation coefficients about the living biomass in term $E_{\mathrm{S}}$ \\
\hline$\epsilon_{\mathrm{Esy}_{1}}$ & 1.0 & exponential attenuation coefficients about the soil moisture of surface layer in term $E_{\mathrm{s}}$ \\
\hline$\phi_{\mathrm{rs}}$ & 0.6 & the parameter describing the vegetation-soil interaction \\
\hline$\kappa_{\mathrm{Er}}$ & 1.0 & attenuation coefficients about the living biomass in term $E_{\mathrm{r}}$ \\
\hline$\epsilon_{\text {Erx }}$ & 1.0 & exponential attenuation coefficients about the living biomass in term $E_{\mathrm{r}}$ \\
\hline$\epsilon_{\mathrm{Ery}_{2}}$ & 1.0 & exponential attenuation coefficients about the soil moisture of root zone in term $E_{\mathrm{r}}$ \\
\hline$\lambda_{\mathrm{rs}}$ & 0.015 & coefficients about precipitation loss due to surface layer in term $R_{\mathrm{S}}$ \\
\hline$\epsilon_{\mathrm{Rsz}}$ & 1.0 & exponential attenuation coefficients about the wilted biomass in term $R_{\mathrm{S}}$ \\
\hline$\kappa_{\mathrm{Rs}}$ & 0.4 & coefficients about the living biomass in term $R_{\mathrm{S}}$ \\
\hline$\epsilon_{\mathrm{Rsx}}$ & 0.7 & exponential attenuation coefficients about the living biomass in term $R_{\mathrm{S}}$ \\
\hline$\epsilon_{\mathrm{Rsy}_{1}}$ & 1.0 & exponential attenuation coefficients about the soil moisture of surface layer in term $R_{\mathrm{S}}$ \\
\hline$\lambda_{\mathrm{Rr}}$ & 0.015 & coefficients about precipitation loss due to surface layer in term $R_{\mathrm{r}}$ \\
\hline$\varepsilon_{\text {Rry }}$ & 1.0 & exponential attenuation coefficients about the soil moisture of root zone in term $R_{\mathrm{r}}$ \\
\hline$\kappa_{\mathrm{Rr}}$ & 0.7 & coefficients about the living biomass in term $R_{\mathrm{r}}$ \\
\hline$\varepsilon_{\mathrm{Rrx}}$ & 0.7 & exponential attenuation coefficients about the living biomass in term $R_{\mathrm{S}}$ \\
\hline
\end{tabular}

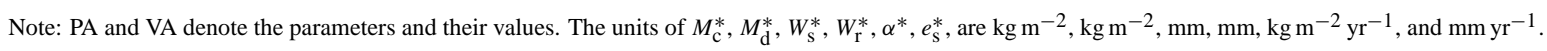

$$
\begin{aligned}
R_{\mathrm{r}}= & \lambda_{\mathrm{Rr}} P_{\mathrm{r}}\left(e^{\varepsilon_{\mathrm{Rry}} W_{\mathrm{r}} / W_{\mathrm{r}}^{*}}-1\right) \\
& {\left[1-\kappa_{\mathrm{Rr}}\left(1-e^{-\varepsilon_{\mathrm{Rrx}} M_{\mathrm{c}} / M_{\mathrm{c}}^{*}}\right)\right] . }
\end{aligned}
$$

The moisture index $\mu$ is obtained by dividing by the dimensional parameters $e_{\mathrm{s}}^{*}$ in the two sides of the Eqs. (A4) and (A5) to also obtain the nondimensionalized grassland model. Thus, the parameter $\mu$ could be obtained. In previous studies, the parameter $\mu$ was fixed on a constant (Zeng et al., 2006; Sun and $\mathrm{Mu}, 2009$ ). However, in this study, the parameter $\mu$ is superimposed on a perturbation, which maybe different each year, as in in every model year during the integration of the five-variable ecosystem model to represent the climate change. The parameters of the equation terms regarding the five-variable grassland model are shown in Table A2.

\section{Appendix B}

\section{The moisture index in northern China}

To quantify the amplitude of the parameter perturbation related to the moisture index, and to make the perturbed moisture index reach the reasonable range, the moisture index is introduced according to the study of Liu and Gao (2008). They employed an index $A$, which was the same as the moisture index $\mu$ in our study, to analyze the variation in the moisture index in northern China. The definition of the index $A$ is the annual precipitation $(P)$ divided by the annual evaporation (EPT), namely,

$A=P / \mathrm{EPT}$.

For $P$ and EPT, they employed the meteorological data of 218 stations from the National Meteorological Information Center regarding the annual precipitation and the annual evaporation in northern China during 1961-2005. They found that the moisture index ranged from about 0.11 to 0.48 
Table B1. The minimum and maximum moisture indexes given by Liu and Gao (2008) in different decades from 1961 to 2005.

\begin{tabular}{cc}
\hline Decade & Moisture index \\
\hline $1961-1970$ & $0.13-0.42$ \\
$1971-1980$ & $0.11-0.45$ \\
$1981-1990$ & $0.12-0.48$ \\
$1991-2000$ & $0.13-0.48$ \\
$2001-2005$ & $0.12-0.44$ \\
\hline
\end{tabular}

during 1961-2005 in northern China (Table B1). However, the annual moisture index was not shown in their studies. The average values of the moisture index in different decades were similar. They also did not show how to quantify $\delta_{2}$. In our study, the choice of $\delta_{2}$ is based on their studies. Next, we will introduce how to obtain the $\delta_{2}$. We will implement a tentative method. The principle of choosing $\delta_{2}$ is as follows. We have known the reasonable range of the moisture index. So, if the perturbed moisture index, which is the annual perturbation being superimposed on the reference moisture index, locates in the reasonable range of the moisture index computed by the meteorological data, we consider that the $\delta_{2}$ could be applied in our study. There are lots of $\delta_{2}$ satisfying the above need. In our study, $\delta_{2}=0.26$, and 0.35 were chosen.

Acknowledgements. The authors thank the reviewers and editors for their valuable suggestions. Funding was provided by grants from National Natural Science Foundation of China (No. 40905050), the KZCX3-SW-230 of the Chinese Academy of Sciences (CAS), LASG Free Exploration Fund, and LASG State Key Laboratory Special Fund.

Edited by: O. Talagrand

Reviewed by: three anonymous referees

\section{References}

Barclay, A., Gill, P. E., and Rosen, J. B.: SQP methods and their application to numerical optimal control, Variational Calculus, Optimal Control and Applications, edited by: Schmidt, W. H., Heier, K., Bittner, L., and Bulirsch, R.: Birkhäuser Verlag, Basel, 207-222, 1998

Chen, H., Tian, H., Liu, M., Melillo, J., Pan, S., and Zhang, C.: Effect of land-cover change on terrestrial carbon dynamics in the southern USA, J. Environ. Qual., 35, 1533-1547, 2006.

Fay, P. A., Kaufman, D. M., Nippert, J. B., Carlisle, J. D., and Harper, C. W.: Changes in grassland ecosystem function due to extreme rainfall events: implications for responses to climate change, Glob. Change Biol., 14, 1600-1608, 2008.

Gao, Q., Li, X. B., and Yang, X. S.: Responses of vegetation and primary production in North-South Transect of Eastern China to global change under land use constraint, Acta Bot. Sin., 45, 1274-1284, 2003.
Gibbard, S., Caldeira, K., Bala, G., Phillips, T. J., and Wickett, M.: Climate effects of global land cover change, Geophys. Res. Lett., 32, L23705, doi:10.1029/2005GL024550, 2005.

Guo, L., Xiong, L., and Wang, W.: Influence of climatic change on Talatan lawn desertification in recent 50 years, Research of Soil and Water Conservation, 15, 57-63, 2008 (in Chinese).

Houghton, R. A. and Hackler, J. L.: Sources and sinks of carbon from land-use change in China, Global Biogeochem. Cy., 17, 1034, doi:10.1029/2002GB001970, 2003.

Liu, J. H. and Gao, J. X.: Spatial changes of boundary based on land use and cliamte change in the farming-pastoral ecotone of northern China, China Environmental Science, 28, 203-209, 2008 (in Chinese)

McGuire, A. D., Sitch, S., Clein, J. S., Dargaville, R., Esser, G., Foley, J., Heimann, M., Joos, F., Kaplan, J., Kicklighter, D. W., Meier, R. A., Melillo, J. M., Moore III, B., Prentice, I. C., Ramankutty, N., Reichenau, T., Schloss, A., Tian, H., Williams, L. J., and Wittenberg, U.: Carbon balance of the terrestrial biosphere in the Twentieth Century: Analyses of $\mathrm{CO}_{2}$, climate and land use effects with four processbased ecosystem models, Global Biogeochem. Cy., 15, 183-206, doi:10.1029/2000GB001298, 2001.

Mitchell, S. W. and Csillag, F.: Assessing the stability and uncertainty of predicted vegetation growth under climatic variability: northern mixed grass prairie, Ecol. Model., 139, 101-121, 2001.

$\mathrm{Mu}, \mathrm{M}$. and Wang, B.: Nonlinear instability and sensitivity of a theoretical grassland ecosystem to finite-amplitude perturbations, Nonlin. Processes Geophys., 14, 409-423, doi:10.5194/npg-14409-2007, 2007.

Mu, M., Duan, W., Wang, Q., and Zhang, R.: An extension of conditional nonlinear optimal perturbation approach and its applications, Nonlin. Processes Geophys., 17, 211-220, doi:10.5194/npg-17-211-2010, 2010.

Rogiers, N., Conen, F., Furger, M., Stöckli, R., and Eugster, W.: Impact of past and present land-management on the C-balance of a grassland in the Swiss Alps, Glob. Change Biol., 14, 26132625, 2008.

Solomon, D., Lehmann, J., Kinyangi, J., Amelung, W., Lobe, I., Pell, A., Riha, S., Ngoze, S., Verchot, L., Mbugua, D., Skjemstad, J., and Schäfer, T.: Long-term impacts of anthropogenic perturbations on the dynamics and speciation of organic carbon in tropical forest and subtropical grassland ecosystems, Glob. Change Biol., 13, 511-530, 2007.

Sun, G. D. and Mu, M.: Nonlinear feature of the abrupt transitions between multiple equilibria states of an ecosystem model, Adv. Atmos. Sci., 26, 293-304, doi:10.1007/s00376-009-02938, 2009.

Vitousek, P. M., Mooney, H. A., Lubchenco, J., and Melillo, J. M.: Human Domination of Earth's Ecosystems, Science, 277, 494499, 1997.

White, T. A., Campbell, B. D., Kemp, P. D., and Hunt, C. L.: Sensitivity of three grassland communities to simulated extreme temperature and rainfall events, Global Change Biol., 6, 671-684, 2000.

Xu, D., Kang, X., Liu, Z., Zhuang, D., and Pan, J.: Assessing the relative role of climate change and human activities in sandy desertification of Ordos region, China, Science in China Series D: Earth Sciences, 52, 855-868, doi:10.1007/s11430-009-0079-y, 2009. 
Zeng, N. and Neelin, J. D.: The role of vegetation-climate interaction and interannual variability in shaping the African Savanna, J. Climate, 13, 2665-2670, 2000.

Zeng, X. D., Zeng, X. B., Shen, S. S. P., Dickinson, R. E., and Zeng, Q. C.: Vegetation-soil water interaction within a dynamical ecosystem model of grassland in semi-arid areas, Tellus B, 57, 189-202, 2005.
Zeng, X. D., Wang, A. H., Zeng, Q. C., Dickinson, R. E., Zeng, X. B., and Shen, S. S. H.: Intermediately complex models for the hydrological interactions in the atmosphere-vegetation-soil system, Adv. Atmos. Sci., 23, 127-140, 2006.

Zhao, H., Okuro, T., Zhou, R., Li, Y., Zuo, X., and Huang, G.: Effects of Human Activity and Climate Changes on Vegetation in Horqin Sandy Grassland, InnerMongolia (in Chinese), Adv. Earth Sci., 23, 408-414, 2008. 